(2) The urgent necessity of the production of food statistics and nutritional data, and the carrying out of national food consumption surveys is stressed.

(3) Special reference is made to the great importance of developing the fishery industries in Pakistan.

(4) The importance of industrial food scientists to establish food manufacturing industries to increase the efficiency of food preservation and distribution nationally, and to foster export trade, is indicated.

(5) The shortage of trained food scientists is a serious retarding factor in the development of food research and industry, and the urgent need of facilities for overseas training of a large body of potential food scientists and technologists is stressed.

\title{
REFERENCES
}

Corran, J. W. (1959). Report to the Government of Pakistan on Organization and Development of Food Research and Technology in the Laboratories of the Pakistan Council of Scientific and Industrial Research. Rome: Food and Agriculture Organization of the United Nations (ETAP).

FAO (1954). F.A.O. nutr. Stud. no. I (revised).

FAO (1956). Yearb. Fish. Statist. 5, A-4.

Fidler, J. C. (1957). Report to the Government of Pakistan on Food Sections of the Region Laboratories, Pakistan Council of Scientific and Industrial Research. Rome: Food and Agriculture Organization of the United Nations (ETAP).

Government of Bengal: Nutrition Committee (1940). Report of the Nutrition Committee.

Nazir Ahmad (195I). Proc. Indo-Pacif. Fish. Coun. sect. II.

Nazir Ahmad (1956). Pakist. F. Sci. 8, no. 4, p. I67.

Nazir Ahmad (1957). Presidential address, section of Biology, Zoology, Botany and Entomology, 9th Pakistan Science Conference, Peshawar, Lahore: Pakistani Association for the Advancement of Science, Ismail Aiwan-I-Science.

Qureshi, M. R. (195I). Pakist. med.F. 7, no. 2, p. 21.

\section{Nutritional change: some comments from social research}

\author{
By A. T. M. Wilson, Unilever House, Blackfriars, London, E.C.4
}

This Symposium has raised in my mind a number of questions and comments touching on areas of interest to those concerned with research in the social sciences. It will be convenient to group my remarks under three headings : first, comments on some points raised during the Symposium; second, a brief and familiar outline of the ideal situation for development of nutritional work; and, thirdly, comments on some persistent difficulties which arise.

\section{Comments on some points raised}

In his opening remarks the Chairman (Aykroyd, I96r) emphasized the similarities as well as the differences in problems of fostering nutritional change in different regional and communal settings. Both are important when we come to consider the practical problems of implementing nutritional programmes; but it is in applying 
knowledge that the social as well as the biological sciences begin to appear as disciplines from which a significant contribution may be needed. As to differences between communities, in discussions of difficulties in more distant countries, I have developed a habit of trying to recall some British or North American equivalent of some particular problem; and descriptions given in the Symposium are likely to have produced a similar response from many of us here.

A second point which has been repeatedly illustrated is the difficulty of integrating the interests and approach of producers on the one hand and consumers on the other. In the background of this problem lie familiar conflicts not only between community groups, but between departments of governments and indeed between international agencies. Even at the level of the household the problem appears in terms of the existence of conflicting as well as common interests in those concerned with housewifery and with husbandry. In some of the comments made during the Symposiumalthough I may be exaggerating this-it seemed to me that there was a strain of exasperation over these conflicts of interest. On the one hand producers, and particularly farmers, have a long, widespread and international history of evoking such feelings; equally, consumers often appear to be unwilling to accept advice and changes which others regard as self-evidently beneficial and based on incontrovertible scientific work.

Two points came up which illustrate some aspects of nutritional problems of concern to the social research worker: first, a familiar situation which can appear in so many forms - in this connexion, that of a mother's taking infants off milk because of gastro-intestinal symptoms and so increasing the chances of kwashiorkor, a situation in which the traditional treatment of a particular sign was dangerous through misinterpretation of the significance of the symptoms. The point I want to stress is that in such traditional treatment by mothers there is usually a logical basis of some kind; the result is not simply due to stupidity or to a careless random action.

Secondly, a point of a general kind has been brought out in different cultures and regions of the world; for example, the same domestic animal can be treated in any or all of a series of ways - as wealth, or as a means of exchange, or as a dowry, as an object of worship or as a source of physical power or, finally, as a source of food. The significance of these very different ways of looking at the same animal in different cultural and social contexts underlines the need to approach nutritional problems on a rather wide basis, rather than from the point of view of any single starting point, be it anthropological, psychological, technological or biochemical.

\section{Some factors in the ideal situation for nutritional programmes}

Ideally, I suppose, a nutritional policy and programme at national level needs a government which is fully cognizant of the wide significance of nutrition and of the very high yield which nutritional investments can give in the health of a community as well as in the health of a people. Where this situation exists, the government concerned would no doubt be advised by a fully acceptable and appropriately structured technical advisory group whose recommendations the central executive would put 
into action as an integrated plan of food production (including exports and imports), of food supply and of nutritional education. Those concerned with the production, supply and distribution of food would, in their turn, have been successful in reconciling the many different interests concerned in the mixed economies which are now usual; and they would have resolved the differences of approach in the contributions made to planning by the relevant technical and professional groups and, indeed, by the pressure groups which usually exist around them. In terms of nutritional education, a substantial, continued, flexible, and successful programme would have led to an adequately high level of knowledge and of nutritional skill in the population as a whole; and there would be a clear awareness of the channels and methods of communication--including the informal opinion-leadership which is so importantby which necessary changes in food production could be adequately reflected by changes in consumption without damage to health and satisfaction.

Finally, all these matters would, in different ways, be the continuing concern of organizations concerned with nutritional research, with development of technical and educational methods and with the training of the relevant staff for work at community and household level. In general terms, this set of agreed ideals has been in existence for something like a quarter of a century, perhaps longer. What are the persistent difficulties of carrying it out in practice?

\section{Some questions about the slow growth of nutritional programmes}

In what follows, the questions raised are likely to be familiar, but if only because of their persistence they are at least worth continuous consideration and discussion.

(I) In setting up a national nutritional programme, or in setting up a nutritional project, what is the status of food science in the experience and knowledge of the relevant community, of its relevant key groups, and of the relevant governmental officials?

The answer to this question would, no doubt, vary greatly as between one community and another. Moreover, nutrition has to compete with many other governmental and community interests; and a considerable public-rel tions programme is likely to be needed on the part of those who are concerned with fostering the growth of nutritional knowledge and advancing nutritional practice if adequate movement is to be maintained in these fields.

(2) In relation to a nutritional policy or project, how should the appropriate technical advisory group be structured and how can it best be related to governmental and community organization so that it can effectively perform its task?

There are many problems in this area: for example, contending vested interests and the problems of interdisciplinary relations either in committee or within project teams.

One of my former colleagues has frequently remarked that 'God has not seen fit to divide up human problems into the same categories as University chairs'. This particular issue is, of course, highly relevant to nutrition, an area of work in which many disciplines are of necessity concerned. If I appear a little too tactful in recording 
the difficulties which social science members can produce in project teams, I hope I will be allowed to say, less indirectly, that members of almost any single discipline are likely to find similar difficulties in working within a mixed team. In the annual report of the Russell Sage Foundation (I959) one particular relevant finding is this: the incidence of interpersonal difficulty in using members or advisers from the social sciences in teams mainly composed of administrators was so high that, to put it bluntly, it was highly improbable that it could be explained on grounds of the types of personalities concerned. The chances that so many awkward people could have been brought together even by active planning were negligible. The conclusion $I$ want to draw is that, almost invariably, there will be complex and difficult problems of team design and organizational planning when scientific specialists of any kind need to work together, either within an organization or in project teams.

(3) How can any wider problems arising from purely nutritional solutions of nutritional problems be foreseen and avoided?

If I may briefly outline the difficulty here, it seems to be analogous to a situation in which, in medical treatment, a drug which seems entirely appropriate to, let us say, a heart specialist in treating some cardiac condition turns out in the longer term to have what are called unfortunate side-effects on, shall we say, the central nervous system. Similarly-the point is, perphaps, too obvious to need any illustrationmany excellent project efforts both in and out of the nutritional field have in the past been found to lead in the longer term to unfortunate consequences in fields outside the immediate interest of those concerned. I put this forward not as an excuse for inactivity but only as a basis for effective interdisciplinary planning at early stages of projects and programmes.

(4) In what circumstances are different kinds of pressure to change nutritional patterns justified?

Dr R. C. Burgess (1956) of the WHO Nutritional Unit has said 'When we inquire into the reason for the all too frequent disregard of local foods we soon find ourselves in the baffling maze of tradition, taboo and magic and of the whole system of belief of the people about health and the causes of disease. In the past most workers have tried, by repeated blows of didactic teaching, to hammer their way through this maze, and break down the resistances to unaccustomed foods and eating practiceswith very little success. More recently, we have come to realize that beliefs about food are part of the whole fabric of the life of a people and cannot be changed in isolation, and that the feeding customs which have become established through centuries of trial and error have developed as part of the people's reaction to the total environment in which they have learned to survive'. Such a statement leads one to consider the nature of nutritional patterns and nutritional change, and to consider the conditions in which learning will be possible; for this is often a better approach than that of considering what kind of instruction needs to be given. Among the important conditions for effective learning, a high place must be given to the condition that those who are being invited to learn have some interest, some motive, to do so, and can both see and fully accept that, if they learn, it will help them to satisfy some felt need. Similarly, another important condition is that those who seek to 
teach should be completely trusted by those who are going to learn; indeed, in most problems of nutritional change it will be necessary for those who are seeking to foster change by educational methods somehow to have earned the right to be trusted by those whom they seek to influence. In the background of many projects which one may read about, it is clear that notions of instruction have had a very prominent place; but from all we know, both in practice and in experiment, while instruction may lead to modest immediate results, on the whole it produces rather poor results in the middle and longer term. On the other hand, educational processes that have been based on the active interest and felt needs of those who are learning, and where the education has been conducted by those who have given good reason to be trusted as teachers, have often enough been shown to have quite definite and persistent results.

Nutrition workers sometimes adopt a rather pessimistic attitude towards what can be done by education, which is not difficult to understand, taking the usual circumstances into account; but it must also be noted that quite dramatic changes in nutritional patterns are not unknown. Some of these have been noted rather than studied or induced; some appear to have been in relation to commercial products. In neither situation, therefore, have they, as a rule, been fully reported in the scientific and technical literature.

(5) What kind of research needs to be undertaken if adequate nutritional policies and programmes are to be brought into being?

There is little doubt that all-round nutritional knowledge could, with advantage, be developed; but there is, equally, little reason to doubt that much of the knowledge we already possess is not being effectively applied. In part this is due, I believe, to the slowness with which social science research in this field has developed; and, although I must declare a bias, I feel that if there are any particularly urgent research needs I would include among them study of the social and cultural aspects of nutritional change. The problems here are not only wide ranging, but have considerable depth and complexity; and if an example is needed I would nominate the remarkable way in which breast-feeding customs appear to be changing in many societies and cultures throughout the world. This problem is commonly considered in some relatively narrow way; but it would be helpful, and may sometimes be essential, to have a much wider framework for study of changes in breast-feeding. The problem itself runs off into the area of powerful and conflicting trends which relate to the role and social status of the mother and the wife and, indeed, to the role of men and women in different types of cultural setting.

\section{REFERENCES}

Aykroyd, W. R. (1961). Proc. Nutr. Soc. 20, 9 1.

Burgess, R. C. (1956). Proc. Nutr. Soc. 15, 13.

Russell Sage Foundation. (1959). Annual Report. New York: Russell Sage Foundation. 